\title{
Enhancement of parity and time invariance violation in Radium atom
}

\author{
V.V. Flambaum * \\ School of Physics, University of New South Wales, Sydney 2052, Australia
}

\begin{abstract}
There are several factors which lead to a huge enhancement of parity and time invariance violating effects in the Ra atom: very close electronic levels of opposite parity, the large nuclear charge $\mathrm{Z}$ and the collective nature of T,Podd nuclear moments. Experiments with Radium may be used to measure it's nuclear anapole, magnetic quadrupole and Schiff moments. Such measurements provide information about parity and time invariance violating nuclear forces and electron-nucleon interactions.
\end{abstract}

*email address: flambaum@newt.phys.unsw.edu.au 
Effects of time invariance $(\mathrm{T})$ and parity $(\mathrm{P})$ violation are inversely proportional to the distance between opposite parity energy levels. There is a pair of close opposite parity levels in the Ra atom, the state $|1>=| 7 s 6 d J^{P}=2^{+}>$, with $\mathrm{E}=13993.97 \mathrm{~cm}^{-1}$ and the state $|2>=| 7 s 7 p J^{P}=1^{-}>$, with $\mathrm{E}=13999.38 \mathrm{~cm}^{-1}$, which are separated by a very small interval $\left(5 \mathrm{~cm}^{-1} \sim 10^{-3} \mathrm{eV}\right)$. Note that there are also very close levels of opposite parity in rare-earth atoms (e. g. in Dy atom [1,2]) which have been used to measure parity violation. The advantage of Ra is that the electron states here are simple. Therefore, the mixing of these states by the weak interactions is not suppressed and can be accurately calculated (contrary to the mixing in the rare-earth atoms where there is a strong suppression of the matrix elements due to the extreme complexity of the electronic eigenstates). There are also extra factors of enhancement in Ra: the large value of the nuclear charge, $Z=88$ (the $\mathrm{P}$ and $\mathrm{T}$ odd effects increase with $\mathrm{Z}$ faster than $Z^{2}$ ) and the collective T,P-odd moments of deformed Ra nuclei that are much greater than the same moments in spherical nuclei [3, This makes Radium an attractive object for future experiments.

The close electron levels in Ra have different electron angular momenta, $\mathrm{J}=1$ and $\mathrm{J}=2$. The conservation of the total angular momentum $\mathbf{F}=\mathbf{J}+\mathbf{I}$ requires the involvement of the nuclear spin $I$ for these states to be mixed. Parity violating effects in this case can be produced by the nuclear anapole moment, which is directed along the nuclear spin. An atomic electric dipole moment ( EDM) appears due to the interaction between the atomic electrons and nuclear T,P-odd moments: magnetic quadrupole, Schiff and electric octupole moments. These effects also can appear due to P, T-odd nuclear-spin-dependent electronnucleus interactions.

Let us start from an estimate of the contribution of the nuclear magnetic quadrupole moment $M$. Atomic EDM in the metastable state $\mid 1>$ appears due to mixing with the opposite parity state $\mid 2>$ by the Hamiltonian $H_{M}$ of the magnetic interaction between the nuclear magnetic quadrupole moment and the atomic electrons. The atomic EDM is given by the following formula

$$
\mathbf{d}=2 \frac{<1\left|H_{M}\right| 2><2|-e \mathbf{r}| 1>}{E_{1}-E_{2}}
$$

The spin-orbit interaction in Radium is very large, therefore the electronic states can be approximately described by jj-coupling. In this case $|1>\simeq| 7 s 6 d_{3 / 2}, J=2>$ and $\mid 2>\simeq$ $\mid 7 s 7 p_{1 / 2}, J=1>$. An expression for the matrix elements of $H_{M}$ between the electronic orbitals $6 d_{3 / 2}$ and $7 p_{1 / 2}$ can be found in [5]. Using this expression and simple numerical estimate for the matrix element of the radius vector $\mathbf{r}$ between these orbitals we obtain the following value for the Ra atomic EDM:

$$
d \simeq 0.5 \cdot 10^{3} M m_{e}
$$

where $m_{e}$ is the electron mass. The magnetic quadrupole moment $M$ is not zero in those $\mathrm{Ra}$ isotopes that have nuclear spin $I \geq 1$, for example, in ${ }^{223} R a$ where $I=3 / 2$. This nucleus is deformed, therefore, it has a collective magnetic quadrupole moment $M$, which was estimated in Ref. [3] to be $M \simeq 10^{-19} \mathrm{~cm} \cdot \eta e / m_{p}$, where $m_{p}$ is the proton mass and $\eta$ is the dimensionless constant of the T,P-odd nucleon-nucleon interaction (the strength of the $\mathrm{T}, \mathrm{P}$-odd interaction is measured in units of the weak interaction Fermi constant). The final result for the atomic EDM produced by the T,P-odd nucleon-nucleon interaction is 


$$
d \simeq 2 \cdot 10^{-20} \eta \text { ecm }
$$

This value is about $3 \cdot 10^{4}$ times larger than EDM of $\mathrm{Hg}$ atom, which was measured in Ref. [6] and gives the present best limit on $\eta$.

The atomic EDM can also be produced by the nuclear Schiff moment (see, e. g. [5]). However, the Schiff moment produces a contact interaction only (an electric field inside the nucleus). The mixing of $6 d_{3 / 2}$ and $7 p_{1 / 2}$ states by this interaction is very small. However, one can take into account configuration mixing and use the maximal matrix element between the $7 s$ and $7 p_{1 / 2}$ states (for example, include mixing between the $7 s 6 d$ and $7 p 7 p$ configurations). Also, there is theoretical and experimental evidence that the odd Radium isotopes have octupole deformation, which leads to a huge $10^{3}$ enhancement of the nuclear Schiff moment [4]. A rough estimate of the Schiff moment contribution that includes this enhancement gives a value of the atomic EDM comparable to the magnetic quadrupole contribution (3). The advantage of the Schiff moment is that it exists also for the isotopes with nuclear spin $\mathrm{I}=1 / 2$, like ${ }^{225} \mathrm{Ra}$ (the magnetic quadrupole is equal to zero for such isotopes).

Finally, Radium atom can be used to measure the nuclear anapole moment. In principle, there are several possibilities. One can measure, for example, interference between the Stark and parity violating amplitudes in the transition between the ground state $|0>=| 7 s^{2}, J=$ $0>$ and excited state $|1>=| 7 s 6 d J^{P}=2^{+}>, E=13993.97 \mathrm{~cm}^{-1}$. The parity violating amplitude $E 1_{p v}$ appears due to the weak mixing between the state $\mid 1>$ and the opposite parity state $|2>=| 7 s 7 p J^{P}=1^{-}>, E=13999.38 \mathrm{~cm}^{-1}$. Again, one has to take into account the configuration mixing since the direct matrix element $<6 d_{3 / 2}\left|H_{a}\right| 7 p_{1 / 2}>$ of the interaction $H_{a}$ between the atomic electrons and the anapole magnetic field which is localized inside the nucleus, is very small. A rough numerical estimate shows that due to the closeness of the opposite parity levels and the large nuclear charge $\mathrm{Z}$ the amplitude $E 1_{p v}$ in $\mathrm{Ra}$ is several hundred times larger than similar amplitude (induced by the anapole) in Cs; the latter amplitude was recently measured in Ref. [7].

Note that in Ra the anapole-induced amplitude gives the dominating contribution to the total parity violating amplitude. This may be an advantage since in Cs the anapole contribution had to be separated from the weak charge contribution ( due to Z-boson exchange between electrons and nucleus) which was two orders of magnitude larger than the anapole contrubution. We may neglect the weak charge contribution in Ra since the states $\mid 1>$ and $\mid 2>$ have different electron angular momenta $\mathrm{J}=2$ and $\mathrm{J}=1$ and cannot be mixed by the weak nuclear-spin-independent interaction.

In conclusion, we considered strongly enhanced parity and time invariance violating effects in Ra atom. Unfortunately, all odd Radium isotopes are unstable. However, recent progress in trapping of unstable elements makes such experiments feasible. For example, an experiment on parity violation in the short-lived Fr atom is in progress 8 . An experiment with ${ }^{225} R a$, motivated by the work [4], has been discussed by S. Lamoreaux [9] and A. Young [10]. The Los Alamos laboratory has a source of this isotope. Note also that the electronic structure of Radium atom is relatively simple (two electrons above closed shells). Our experience has shown that the accuracy of atomic calculations for such systems can be about one per cent (see, e.g. [11]).

This work was supported by the Australian Research Council. 


\section{REFERENCES}

[1] V.A.Dzuba, V.V. Flambaum, I.B. Khriplovich. Z. Phys. D1, 243, 1986.

[2] A.T. Nguyen, D. Budker, D. DeMille, and M. Zolotorev. Phys. Rev. A56, 3453 (1997).

[3] V. V. Flambaum, Phys. Lett. B 320, 211 (1994).

[4] N. Auerbach, V. V. Flambaum, and V. Spevak, Phys. Rev. Lett. 76, 4316 (1996). V. Spevac, N. Auerbach, and V.V. Flambaum, Phys. Rev. C 56, 1357 (1997). V.V. Flambaum, D. W. Murray and S.R. Orton, Phys. Rev. C 56, 2820 (1997).

[5] O.P. Sushkov, V.V. Flambaum, and I.B. Khriplovich. Zh. Exp. Teor. Fiz. 87, 1521 (1984). [Sov. Phys. JETP 60, 873 (1984)].

[6] J.P. Jacobs, W.M. Klipstein, S.K. Lamoreaux, B.R. Heckel, and E.N. Fortson. Phys. Rev. A52, 3521 (1995).

[7] C.S. Wood, S.C. Bennet, D. Cho, B.P. Masterson, J.L. Roberts, C.E. Tanner, and C.E. Wieman. Science 275, 1759 (1997).

[8] W.Z. Zhao, J.E. Simsarian, L.A. Orozco, W. Shi, and G.D. Sprouse, Phys. Rev. Lett. 78,4169 (1997).

[9] S. Lamoreaux. Talk at the Second International Symposium on Symmetries in Subatomic Physics, Seattle, June 25-28 (1997).

[10] A. Young, private communication.

[11] V. A. Dzuba, V. V. Flambaum, and M. G. Kozlov. Phys. Rev. A 54, 3948 (1996). 